\title{
Linguistic Controller Design of LDS upon Cell-to-cell Mapping
}

\author{
XIAO Zhi-Quan \\ School of Mechanical Engineering and Automation, Wuhan Textile University \\ Wuhan, Hubei, P. R. China \\ e-mail: xzq.power@gmail.com
}

\begin{abstract}
The theory of linguistic dynamic system (LDS) focuses on modeling, analysis, control and evaluation of complex systems at a linguistic level through computing with words. The concepts, frameworks and methods in conventional dynamic systems (CDS) are adopted in research of LDS. This paper is concerned about linguistic controller design of LDS upon cell mapping concept. In view of the studies of controller design for type-I and type-II LDS and optimal fuzzy controller design and tuning methods based on cell-to-cell mapping, two approaches to construct optimal linguistic controller for LDS using cell-to-cell mapping techniques are outlined in this paper.
\end{abstract}

Keywords- Linguistic dynamic system; Cell-to-cell mapping; Optimal fuzzy controller; Controller design; Fuzzy

\section{INTRODUCTION}

To deal with large complex systems effectively with linguistic human knowledge, the theory of linguistic dynamic system is introduced by Wang [1]-[4], which is suitable for modeling, analysis and synthesis of knowledgebased systems, expert systems etc. Since then many efforts have been made on this area. Wang outlined a computational theory for LDS that is computationally feasible by fusing procedures and concepts from different areas: Kosko's geometric interpretation of fuzzy sets, Hsu's cell-to-cell mappings in nonlinear analysis, Zadeh's computing with words, dynamic programming in optimal control theory etc[5]. The basic research contents, research methods and computational framework of LDS are proposed. As proposed in [6], one special kind of LDS is fuzzy dynamic systems. Two types of fuzzy dynamic systems, which are then regarded as special case of type-I LDS and type-II LDS, can be generated form CDS by means of fuzzy extension principle and fuzzy composition, respectively. The theory and design methods, characteristics such as stability, fixed points of type-II LDS, are studied. Furthermore, researches on equilibrium terms, invariant term set, period-doubling bifurcation and chaos behaviors of LDS are carried out [5][8]. Recently, type-2 fuzzy logic is used in modeling, analysis of LDS and in the construction of the so-called word-cell [9]-[13].

A design procedure of linguistic controller of type-II LDS was presented in literature [6], which utilized fuzzy composition and a numerical iteration minimizing the loss of certainty in each iteration. The standard "defuzzifiers" were not needed in this procedure. Another linguistic feedback law design method [5], leading to an optimal linguistic controller, was introduced based on Hsu's discrete method of optimal control and cell-to-cell mappings. In spite of lacking of detailed studies on this method, it is worth further investigation, for it provides a way to construct linguistic controller for LDS based on cell-to-cell mapping generated optimal control data of a system, rather than using data from human experience. Cell-to-cell mapping, introduced by Hsu [14]-[15], is a powerful computational tool for analyzing the global behavior of nonlinear systems. By partitioning a continuous phase space into a finite number of disjoint cells and approximating system trajectories as cell transitions, it can provide global measures of dynamic performance characteristics which are valuable in system analysis and controller design. After Hsu proposed the cell-to-cell mapping method to search for the optimal control law [16], it has caused considerable research interests. Researchers developed various improved approaches that incorporated the cell mapping method into fuzzy controller design and tuning [17]-[24], which are of great reference to linguistic controller design for LDS.

This paper reviews controller design of LDS, the algorithms of fuzzy controller design and tuning through cell-to-cell mapping techniques. On this basis, the outline of linguistic controller design methods for LDS upon cell-tocell mapping is drawn.

\section{REVIEWS ON LINGUISTIC AND OPTIMAL FUZZY CONTROLLERS DESIGN}

\section{A. Controller Design of Type-I and Type-II LDS}

To effectively study LDS, Wang defined two types of LDS systems, type-I LDS and type-II LDS[4].

A special kind LDS is fuzzy dynamic system and can be generated from conventional dynamic systems by using two methods, the fuzzy extension principle that leads to the a special case of type-I LDS, fuzzy compositions that leads to type-II LDS. And more specifically, a type-I LDS system is defined by a linguistic state space and conventional evolving law while both state space and conventional evolving law a type-II LDS system are of linguistic. And the evolving laws of a type-II LDS can be represented as fuzzy relations which comprise a series of rules with the form of "IF-THEN". The antecedent and consequent of the rules are linguistic terms (words). Through the so-called abstracting process, a conventional dynamics system can be converted into a type-I LDS, and then the behaviors of a type-I LDS can be numerically studied with the $\alpha$-cut mapping method. Since the evolving laws of type-I LDS are in the numerical domain, the controller can be directly constructed from its conventional counterpart by using fuzzy extension principle. 
As to the controller design of type-II LDS, if the structure of conventional controller is known, a three-step design procedure is presented to establish a type-II linguistic controller. The three steps include abstracting linguistic model of the plant, abstracting linguistic model of sensors and abstracting linguistic model of actuators. On this basis, numerical procedures of solving type-II LDS controller can be performed. In [6] a similar design procedure of linguistic controller of type-II LDS, together with optimization procedure of the control policy, is introduced, which is also based on fuzzy extension principle. An optimal linguistic controller design method for a LDS using cell-to-cell mapping is outlined in [5]. By introducing cell state spaces on fuzzy hypercube for a LDS, it utilizes all the concepts and methods of cell state mapping, and the design of optimal controller for a LDS is turned into a standard dynamic programming problem with discrete states and control variables.

Although previous researches mainly focus on generating linguistic controller for a LDS by fuzzy extension principle, there are some other ways worth exploring such as data mining, using expert's knowledge and cell mapping based techniques etc.

\section{B. Fuzzy Controller Design Using Cell-to cell Mapping Techniques}

Several cell-state-based methods for designing optimal fuzzy controllers are described in this section.

The design methodology of Vachtsevanos and coworkers[17], [18], [24] is similar to Hsu's approach except that it produces an optimal control table containing fuzzy rules. It partitions both state space and control variable ranges into sub-intervals called "cell-groups". The evolution of cell-groups in a cell map is evaluated by applying a control action (corresponding to the center-point of a cell in the control action space) as input to the system. After all the cell groups in the state space are examined for all possible control actions, the simulation process stops and this yields a large database containing information about the global behavior of the system for all possible inputs. And then a search algorithm is used to form an optimal control table like Hsu's DOC table. The final step is to generate optimal fuzzy control rules from the optimal table entries, which is accomplished by fuzzifying the borders of cells in the state space and control action space. Then linguistic values defined by triangular membership functions with centers located at the center-points of cells and ends located at the center-points of the adjacent cells are employed. The antecedents and consequents of the final fuzzy control rules are defined using the linguistic values for the cell state space and control action cell space, respectively. Due to the introduction of fuzzy interpretation, the designed fuzzy controller improves on the Hsu controller in that it produces smoother outputs with fewer oscillations and steady-state errors. But like the Hsu's optimal controller, the number of rules equals to the number of cell groups, which will result in large number of rules for high-order system.

The Smith-Comer design technique [19]-[20] uses the least-mean-square algorithm to fine-tune a cell space approximation to an optimal control solution. It yields rulebased Takagi-Sugeno fuzzy controller that approximates an optimal control table. This fuzzy controller is superior to Hsu's optimal-table-based controller in that it produces smooth outputs with less oscillations, chattering, and steadystate errors. It requires fewer rules than the Hsu's and Vachtsevanos' controllers [24].

The method of $\mathrm{Hu}$, Tai and Shenoi [11]-[12] directly incorporates cell map information generated by Hsu's unravelling algorithm in a genetic algorithm for tuning Takagi-Sugeno fuzzy controllers. It engages a simple, intuitive cell-map-based objective function for the genetic search. Since the cell map information over the entire region of operation is used as feedback in controller design, the methodology produces high-performance fuzzy controllers with "globally" time-optimal characteristic and maximal stability and controllability [24].

The so-called fuzzy cell-to-cell mapping method presented by Yen and his co-workers relaxes the requirement on the number of cells while in generating a sub-optimal fuzzy controller [22]. It uses the central cell and its neighboring cells for input variables. In application a triangular-type membership function is applied, where the closeness of the location of the state to the cell center defines the membership of the state to the cell. The inference rules are designed as the form of IF-THEN extracted from cell mapping based optimal control law searching procedure. Simulations and experimental results show that, even with coarse cellular structure this method can produce smooth control efforts and eliminates the problem of steady-state error accumulation caused by the inexact state description. The method is extended to the application of a high-order (six order) system, satellite attitude maneuvering and stabilizing control [23].

\section{OUTLINE OF CONSTRUCTION OF LINGUISTIC}

\section{CONTROLLERS OF LDS USING CELL-TO-CELL MAPPING}

According to the previous reviews, if the structure of a conventional dynamic system is known, two approaches to construct optimal linguistic controller for LDS based on cell mapping techniques are outlined below.

One approach, proposed in [5], is to find a linguistic feedback law that optimizes a linguistic performance index on fuzzy cell space (fuzzy hypercube). First a CDS is converted to a type-II LDS using fuzzy compositions. Endowing the LDS with a cellular structure by applying Kosko's interpretation of fuzzy sets and Hsu's cell-to-cell mapping, a fuzzy cell space on fuzzy hypercube is set up. A cell, or called a word cell, is represented by the cell center in the sense that all points in the cell have similar membership functions, considered as a single linguistic term. And then a linguistic performance index is established based on linguistic terms (fuzzy sets) form of states, controls, one-step cost, target etc. Then with cell-to-cell mappings, the design of optimal linguistic controller for a LDS becomes a standard dynamic programming problem with discrete states and control variables. Through a searching algorithms proposed in [25], an optimal control table is founded which provides the optimal control action at each word cell. The linguistic 
controller of LDS appears as the rules extracted from the optimal control table. Fig. 1 shows the diagram of the approach.

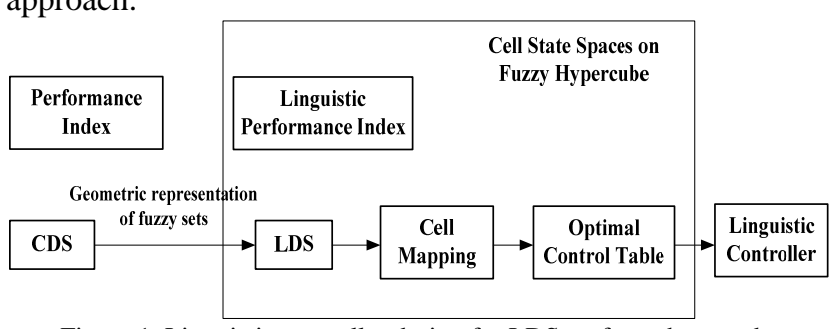

Figure 1. Linguistic controller design for LDS on fuzzy hypercube

The other approach is characterized by adopting cell mapping techniques for construction of optimal controller directly on cell state space comparing to the previous one, shown in Fig.2. It starts with the building-up of a cellular structure for the CDS, and a performance index for control which may be converted from linguistic type of performance index. Optimal control table is generated through cell mapping based searching algorithm. Then different methods can be used to extract linguistic control laws from the optimal control table. Some important issues should be paid attention to during this process: how to mine and design the rules from the numerical information of the optimal cell mapping data, and how to achieve a balance between rule numbers and completeness of the controller. And the resultant linguistic optimal controller is, but is not limited to, in the form of fuzzy controller satisfying the performance index. In addition, some adjustments to the control laws should be taken, in some cases, to accommodate the structure of corresponding LDS. For example, if the antecedents and consequents of the rules extracted from the optimal control table do not corresponds exactly to the state terms and control terms of the LDS, corresponding relationship between them should be established by certain measures. The approach is outlined as follows

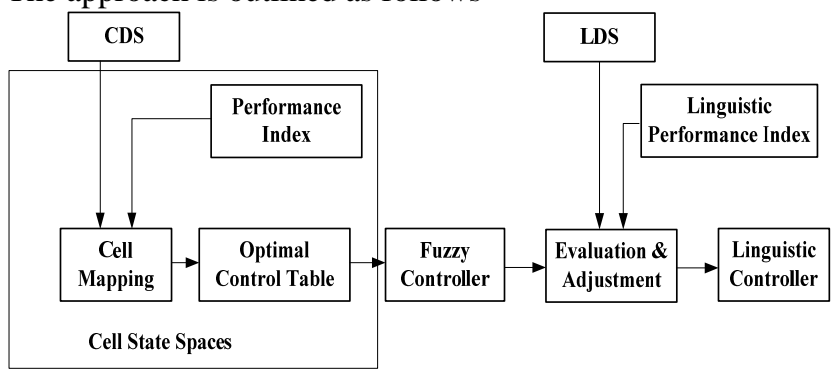

Figure 2. Linguistic controller design for LDS on cell state space

An n-dimensional dynamic system we are considering can be represented by

$$
\dot{x}=f(x, u, t) \quad x \in R^{n}, u \in R^{m}, t \in R^{+}
$$

where $x$ are the states, $u$ the control inputs, and $f$ is a nonlinear mapping.

To construct optimal controller of the dynamic system with cell-to-cell mapping technique, a cellular structure can be introduced by dividing the continuous state space into finite discrete cells. Let $x_{i}, i=1,2, \ldots, n$, be a state variable of the state space, and let the $x_{i}$-axis be divided into a large number of intervals of uniform size $h_{i}$. The interval $z_{i}$ along the $x_{i}$-axis contains all $x_{i}$ satisfying

$$
\left(z_{i}-1 / 2\right) h_{i} \leq x_{i}<\left(z_{i}+1 / 2\right) h_{i}
$$

Thus endowing the state space with the cellular structure, an n-tuple of integers $\left(z_{1}, z_{2}, \cdots, z_{n}\right)$ can be assigned to the cell of the cell space. Let the control output be a mdimensional vector

$$
U=\left[u_{1}, u_{2}, \cdots, u_{m}\right]^{T}
$$

The number of the subintervals for each state variable is chosen in accordance to such considerations: ranges of variables and their resolution or accuracy requirements, computational complexity and implementation time limitations in practical application, the number of rules that are considered manageable in the sense of minimizing the heuristic search time, and the method used in construction of the controller etc. Intervalwise constant control is assumed which means that the control vector remains constant over one time interval. The dynamic system Eq. (1) can be transformed to or approximated by a dynamical system with discrete cell state space

$$
z(k+1)=C(z(k), u(k), t(k)), \quad z \subset Z^{n}, u \in U, t \in T
$$

where $\mathrm{T}$ is the set of all admissible time intervals, and $\mathrm{Z}$ denotes integers. Then the dynamics of the system are expressed in terms of cell-to-cell mappings.

The performance index for the control can be defined by

$$
J=\sum_{j=1}^{n_{c}} \Phi(z(j-1), u(j), t(j))
$$

where $\Phi$ is the one-step cost function. Let $0<w_{1}<w_{2}<\cdots<w_{N w}$ denote all the possible cost increments, where $N_{w}$ is the number of all possible cost increments. Then the cost increment incurred by the $n$th interval control can be expressed as

$$
w(k)=\Phi(z(k-1), u(k), t(k))
$$

Generally, optimization problem can mean minimum time (time-optimal), minimum energy, or minimum error etc. Then general form of cost function can be introduced as

$$
\Phi(z(k-1), u(k), t(k))=\sum \alpha t(k)+\beta u^{2}(k)+\gamma g(k)
$$

where $\alpha, \beta, \gamma$ are coefficients, and $g(k)$ denotes a nonnegative expression for certain physical quantity. For 
example, $g(k)=e 2(k)$ can represent control errors, where $e(k)$ denotes error between output and desired input. For a timeoptimal control problem, the coefficients can be set as $\alpha=1$, $\beta=\gamma=0$.

Next step is to establish the optimal control table with numerical computations in cell state space and searching algorithm. Hsu's original searching method or some modified methods can be used. Once the optimal control table is obtained, a fuzzy controller, which is a kind of linguistic controller, can be constructed through various ways described as above. The controller comprises a number of rules in the form of $\mathbf{I F}<$ antecedent (conditions) $>$ THEN $<$ consequent (control action) $>$.

So far, the construction process of linguistic controller occurs on the cell state space of the CDS. If the linguistic controller obtained can be used directly for the LDS converted from the corresponding CDS needs to be evaluated. Therefore, the final step of the process is to evaluate and adjust the linguistic controller for the LDS. Further studies are needed on this topic.

\section{CONCLUSIONS}

Linguistic controller design for LDS with cell-to-cell mapping method is concerned. The methods of linguistic controller design for LDS have been researched by Wang. And some techniques on optimal fuzzy controller design and tuning based on cell-to-cell mapping have been presented by researchers. Based on the reviews, two approaches to construct optimal linguistic controller for LDS using cell mapping techniques are outlined in this paper. One approach generates linguistic controller for LDS on fuzzy hypercube (fuzzy cell state space) based on the conversion from conventional dynamic system to LDS. For the other approach, an optimal fuzzy controller is first constructed with cell mapping techniques. And then fuzzy controller needs some adjustments for corresponding LDS if necessary.

\section{ACKNOWLEDGMENT}

The author would like to thank the support from the Key Laboratory of Complex System and Intelligent Science, Institute of Automation, Chinese Academic of Science.

\section{REFERENCES}

[1] Wang F.Y. "Modeling, analysis and synthesis of linguistic dynamic systems: a computational theory," Proceedings of IEEE Int'l Workshop on Architecture for Semiotic Modeling and Situation Control in Large Complex Systems, IEEE Press, 1995, pp. 173-178.

[2] Wang F.Y., "Fundamental issues in research of computing with words and linguistic dynamic systems," Acta Auto-matica Sinica, vol.31, 2005, No.6, pp.844-852, (In Chinese)

[3] Wang F.Y., "On the abstraction of conventional dynamic systems: from numerical analysis to linguistic analysis," Information Science, vol. 171, 2005, No. 1-3, pp. 233-259.

[4] Wang F.Y., "Outline of a computing theory for linguistic dynamical systems: towards computing with words," International Journal of Intel. Control and System, vol. 2, 1998, No. 2, 211-224.

[5] Wang F.Y., Hongbin Zhang, "A Computational Approach for Linguistic Dynamic Systems and Its Application to Hybrid Control
Systems," Proceedings of 1996 IEEE International Conference on Systems, Man and Cybernetics, vol.4, 1996, pp. 2613-2618.

[6] Wang F.Y., Tao Yang, "On Linguistic analysis of Numerical Dynamic Systems," Proceedings of the 2002 IEEE International Symposium on Intelligent Control, 2002, pp. 850-855.

[7] Mo Hong, "Linguistic Dynamic Systems Based on Computing with Words and Their Relative Analysis," Ph. D. dissertation, Institute of Automation, Chinese Academy of Sciences, Beijing, 2004, (In Chinese)

[8] Mo Hong, Wang F. Y., "Linguistic dynamic systems based on computing with words and their stabilities" Science in China, vol. 52, 2009, No. 5, pp. 780-796.

[9] Zhao Liang, "Computing with Words Study and Linguistic Dynamic Systems Research Based on Interval Type-2 Fuzzy Sets Method," Ph. D. dissertation, Institute of Automation, Chinese Academy of Sciences, Beijing, 2009, (In Chinese)

[10] Mo Hong, Wang F.Y., Zhao Liang, "LDS trajectories under one-toone mappings in interval type-2 fuzzy sets," Pattern Recognition and Artificial Intelligence, vol. 23, 2010, No. 2, pp.144-147, (In Chinese)

[11] MO Hong, WANG Fei-Yue, XIAO Zhi-Quan, Chen Qian, "Stabilities of Linguistic Dynamic Systems Based on Interval Type-2 Fuzzy Sets," Acta Auto-matica Sinica, vol.37, 2011, No. 8,1-7, (In Chinese)

[12] Xiao Zhi-Quan, Mo Hong, "Word-Cell Based on Type-2 Fuzzy Set," In: Proc. of IEEE International Conference on Computational Intelligence and Software Engineering, 2009, Vol.3.

[13] Xiao Zhi-Quan, Mo Hong, "Preliminary Studies on Word-cell and Its Properties," Proceedings of the 2012 International Conference on Technology and Management (ICTAM 2012), Jun. 2012, pp.237-246

[14] Hsu, C.S, "A theory of cell-to-cell mapping dynamical systems," Journal of Applied Mechanics Trans. of ASME, vol.47, 1980, pp. 931-939

[15] Hsu, C.S, Cell-to-cell mapping. Springer-Verlag, New York, 1987

[16] Hsu, C.S., "A discrete method of optimal control based upon the cell state space concept,". Journal of optimization theory and applications, vol. 46, 1985, No. 4, pp. 547-569.

[17] S.S. Farinwata and G. Vachtsevanos, "Stability of a fuzzy logic controller designed by the phase portrait assignment algorithm," Proceedings of the Second IEEE International Conference on Fuzzy Systems, 1993, pp. 1377-1382.

[18] G. Vachtsevanos, S.S. Farinwata and D.K, "Pirovolou: Fuzzy logic control of an automotive engine. IEEE Control Systems Magazine," vol. 13, 1993, No. 3, pp. 62-68.

[19] Samuel M. Smith, David J. Comer, "Self-tuning of a fuzzy logic controller using a cell state space algorithm," Proceedings of IEEE International Conference on Systems, Man and Cybernetics, 1990, pp.445-450.

[20] Smith, S.M., and Comer, D.J., "Automated calibration of a fuzzy logic controller using a cell state space algorithm," IEEE Control Systems, vol.11, 1991, No. 8, pp. 18-28.

[21] H.T. Hu, H.M. Tai and S. Shenoi, "Incorporating cell map information in fuzzy controller design," Proceedings of the Third IEEE International Conference on Fuzzy Systems, 1994, pp. 394-399.

[22] J.Y. Yen, W.C. Chao, S.S. Lu, "A Fuzzy Cell Mapping Method for a Sub-optimal Control Implementation," Control Engineering Practice, vol. 2, 1994, No. 2, 247-254.

[23] Jia-Yush Yen, Shiou-Wen Tarng, "A fuzzy cell-mapping feedback control algorithm for the satellite attitude maneuvring control," 1996 Asian Fuzzy Systems Symposium, 1996, pp. 567-572.

[24] M. Papa, Hen-Ming Tai, Sujeet Shenoi, "Evaluation of Cell State Techniques for Optimal Controller Design," Proceedings of IEEE International Conference on Fuzzy Systems, 1995, pp.1331-1338.

[25] Wang F.Y., P.J.A. Lever, "A Cell Mapping Method for General Optimum Trajectory Planning of Multiple Robotic Arms," Journal of Robotic and Autonomous Systems, vol. 12, 1994, No. 4, pp. 15-27. 